\title{
Studying gaze abnormalities in autism: Which type of stimulus to use?*
}

\author{
Ana Saitovitch $^{1 \#}$, Anne Bargiacchi ${ }^{1,2}$, Nadia Chabane ${ }^{1,2}$, Anne Phillipe $^{3}$, Francis Brunelle ${ }^{1}$, \\ Nathalie Boddaert ${ }^{1}$, Yves Samson ${ }^{4}$, Monica Zilbovicius ${ }^{1}$ \\ ${ }^{1}$ U1000 Brain Imaging in Psychiatry, INSERM-CEA, Pediatric Radiology Service, Necker Enfants Malades Hospital, Paris V René \\ Descartes University, Paris, France \\ ${ }^{2}$ Child Psychiatry Service, Robert Debre Hospital, Paris, France \\ ${ }^{3}$ U781, Génétique Service, Necker Enfants Malades Hospital, Paris V René Descartes University, Paris, France \\ ${ }^{4}$ Stroke Unity, Pitié-Salpêtrière Hospital, Paris VI Pierre et Marie Curie University, Paris, France

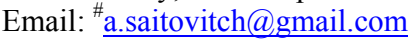

Received 28 February 2013; revised 31 March 2013; accepted 9 April 2013

Copyright (C) 2013 Ana Saitovitch et al. This is an open access article distributed under the Creative Commons Attribution License, which permits unrestricted use, distribution, and reproduction in any medium, provided the original work is properly cited.

\begin{abstract}
Background: Eye-tracking has been used to investigate social perception in autism spectrum disorder (ASD) with variable results. This heterogeneity may be due to the types of stimuli used. In this study, we investigated whether the use of moving vs static stimuli or human actors vs cartoons characters would be more sensitive in detecting gaze abnormalities and discriminating children with ASD from typically developing children. Methods: We studied 18 children with ASD (mean age $=12.9 \pm 2.9$ ) and 21 typically developing controls (mean age $=11.3 \pm 2.5$ ). Gazes were tracked using Tobii-T120 eye-tracker. Four different types of stimuli were presented: movie with human actors, cartoon movie, picture with human actors and cartoon picture. To identify the type of stimuli that best discriminate the ASD group from the control group, a two-way ANOVA was performed using ecological dimension [human-actors/cartoon] and presentation form [movie/picture] as factors. Results: Children with ASD presented significantly less fixations to eyes and faces in the movie with human actors and in the picture with human actors. Children with ASD also presented significantly more fixations to non-social backgrounds in the movie with human actors and in the cartoon movie. A significant ecological effect was observed for the reduction in fixations to the eyes [human-actors > cartoon]. A signifi-
\end{abstract}

\footnotetext{
The authors declare no conflicts of interest in this study.

Funding: Fondation de France, Fondation Orange, PHRC. The funders had no role in study design, data collection and analysis, decision to publish, or preparation of the manuscript.

${ }^{\#}$ Corresponding author.
}

cant presentation form effect was observed for the increased fixations to the non-social background [movie > picture]. Conclusions: The direct comparison of gaze behavior across four different types of stimuli demonstrates that gaze abnormalities in ASD depend on the type of stimuli that is used. Our results suggest that general gaze abnormalities in children with ASD are better detected when using dynamic stimuli, and finer details of these abnormalities, especially looking less to the eyes, are better detected in a more ecologically relevant situation presenting human characters.

Keywords: Autism; Eye-Tracking; Social Perception

\section{INTRODUCTION}

The most common clinical sign of autism spectrum disorder (ASD) is social-interaction impairment, which is associated with verbal and non-verbal communication deficits and stereotyped obsessive behaviors [1,2]. One of the most striking manifestations of social-interaction impairment in ASD is abnormal eye contact during social interactions [3-5].

For several years now, gaze abnormalities can be quantified using eye-tracking technology [6-8]. This methodology has been used to investigate gaze and social perception processes in autism, although the results have been somehow inconsistent. Several studies have described gaze abnormalities in subjects with autism when compared to typically developing controls [9-18], whereas others found no differences between groups regarding gaze patterns $[14,18-20]$. These studies were performed using different populations and a variety of stimuli, which 
may account for the observed heterogenity of the outcomes $[7,21]$.

The first study to use eye-tracking technology in autism, published by Klin and colleagues in 2002 [10], was performed in a group of 15 high-functioning teenagers and adults with ASD. They used scenes from the film Who's Afraid of Virginia Woolf as dynamic stimuli to study differences in gaze pattern. This study showed that subjects with ASD looked to the eyes significantly less than the typically developing controls and looked significantly more to non-social background areas in the scenes.

Subsequent to this work, a number of studies have used eye-tracking methodology to better characterize social cognition in autism [7,21]. For this purpose different strategies were used varying from passive visualization of social scenes to more complex cognitive task performance. Concerning passive visualization, a large variety of stimuli was used including dynamic or static stimuli ranging from human actors to cartoons. Here, we will focus on these passive visualization eye-tracking studies in autism, with emphasis on the characteristic of the stimuli which was presented (movies, pictures, human actors or cartoons).

In a group of children and teenagers with ASD, Speer and colleagues used both dynamic (movies) and static (photos) stimuli created from the film used by Klin and colleagues (Who's Afraid of Virginia Woolf). They described a significant decrease in visual fixation to the eyes in participants with ASD compared with the matched controls but only in a two-character interaction dynamic scene and not in other types of stimuli [14].

Riby and colleagues also used movies as stimuli in an eye-tracking study with children and described that children with ASD looked less to the face of characters and more to regions outside of the social areas than typically developing children regardless of whether the stimulus was a movie containing human actors or cartoons [15]. Interestingly, differences in gaze patterns to the eyes between the two groups were reported only for movies containing human actors. A different study of adults and children with ASD using movies with human actors as stimuli showed that adults with ASD looked significantly less to the eyes than the matched controls did, but no significant differences were found when comparing children with ASD to control children [18]. In opposition to this result, using movies with human actors, Jones and colleagues described that very young children with ASD looked significantly less to the eyes than control children [12]. More recently, a study using a naturalistic interaction paradigm showed that children with ASD looked significantly less and for shorter amounts of time at the face of the adult interacting with them than their typi- cally developing counterparts [22].

In studies using pictures of cartoon characters [19] or pictures of human actors [20], van der Geest and colleagues found no significant differences in gaze patterns between a group of children with ASD and a matched control group. In contrast, a different study, which also presented static pictures of human actors to high-functioning adults with ASD, determined that the adults with ASD looked less to core features of the face (e.g., eyes, mouth and nose) than a group of typically developing controls [16]. This type of result was also described by Riby and colleagues [9] in a study of children using static pictures of human actors. In this study, children with ASD looked significantly less to the eyes than children from the matched control group. In a subsequent study from the same research group, pictures of cartoon characters were used, and children with ASD looked less to the face and more to areas outside of the face than the matched controls did [15].

Thus, although eye-tracking studies of social perception in autism have led to the identification of abnormal behaviors when accessing social information that are characteristic in subjects with autism, the results remain heterogeneous. This heterogeneity is even more evident in studies concerning child populations. One reason for this inconsistency may be that these studies differ widely with respect to the type of stimuli that were used (e.g., movies, pictures, human actors or cartoon characters). This leads to the question of which type of stimuli would be more appropriate for studying social perception in children with ASD. Even though some studies have considered this issue by presenting different types of stimuli to the same subjects, they have obtained their results for each type of stimulus independently but without a direct statistical comparison across stimuli.

Therefore, the objective of this study was to investigate the four main types of stimuli (i.e., movies with human actors, pictures with human actors, cartoon movies and cartoon pictures) usually used in social perception studies in autism to determine the stimulus that is most sensitive for detecting gaze abnormalities and best discriminates children with ASD from typically developing children. To our knowledge, no previous study has directly compared the use of these four types of stimuli, and thus, this study may provide an important methodological foundation for the development of future protocols for studying social perception in autism.

\section{METHODS}

\subsection{Participants}

Thirty-nine children participated in this study. Eighteen children with ASD were recruited in a university hospital 
with dedicated units designated as reference centers for autism by the French Health Ministry. Children were diagnosed according to the DSM-IV [23] and ADI-R [24] criteria for autism, and diagnoses were performed in these units by a multidisciplinary team, including child psychiatrists, child psychologists and speech therapists, during 3 - 7 days of extensive evaluation. Children in the ASD group had a mean chronological age of $12.9(\mathrm{sd}=$ 2.9) and a mean IQ of $93.9(\mathrm{sd}=18.8)$. Twenty-one typically developing children were recruited using an advertisement, and the mean age of this group was 11.3 (sd $=2.5$ ). There was no significant difference between the mean ages in the two groups $(\mathrm{p}=0.28)$. All children had normal or corrected-to-normal vision.

The study was performed in accordance with ethical and legal guidelines. Necker Ethics Committee approved the study and all children had written informed consent of their parents to participate.

\subsection{Stimuli and Settings}

All stimuli were taken from commercial films for children: the Peter Pan ${ }^{\mathrm{TM}}$ film and Peter Pan ${ }^{\mathrm{TM}}$ cartoon. Four different types of stimuli were created: a segment from the movie with human actors, a segment from the cartoon movie, a picture taken from human actors movie and a picture taken from the cartoon movie.

To make possible a direct comparison of the four different stimuli, the duration of the data analyzed for each stimulus was the same (5 seconds). Human actors and cartoon pictures were presented for 5 seconds each and were extracted from the 5 seconds respective movie segments. In order to display an entire social interaction, movie segments where presented for a longer time. The four different stimuli were presented in a randomized order.

These four types of stimuli were used to quantify gaze behavior in children watching social scenes with different presentation forms [movies/pictures] and different ecological dimensions [human actors/cartoon].

The experiment was performed using Tobii T120 Eye Tracker equipment consisting of a 17 -inch TFT monitor with a resolution of $1280 \times 1024$ pixels, on which the stimuli were presented and from which gaze behavior was recorded simultaneously. The eye-tracking system is completely non-invasive with little indication that eye movements are being tracked, and artificially constrain head or body movements are not required. The system tracks both eyes to a rated accuracy of 0.5 degrees with a sampling rate of $60 \mathrm{~Hz}$. The Tobii equipment was connected to a HP Pavillon dv6 laptop computer (Windows 7 Professional) that was used to run the tasks.

The participants were tested individually. The entire session lasted approximately $10 \mathrm{~min}$. The participants were seated facing the eye-tracking monitor at a distance of approximately $60 \mathrm{~cm}$ with the experimenter sitting next to the participant to control the computer without interfering with the viewing behavior. The participant was told they would see different types of movies and pictures. A calibration test consisting of 5 registration points was performed before each set of stimuli. The calibration was repeated if one of the 5 points was not valid. The participants were asked to look at the movies and pictures as they were presented on the screen, and no further instruction was provided. Each participant was debriefed at task completion. Stimulus creation, calibration procedures, data acquisition and visualization were performed using the Tobii Studio ${ }^{\mathrm{TM}}$ Analysis Software.

\subsection{Data Processing and Statistical Analyses}

Data were analyzed using the Tobii Studio ${ }^{\mathrm{TM}}$ Analysis Software. For each of the four stimuli, areas of interest (AOIs) were drawn to investigate fixations to specific regions. Face AOIs were marked with an oval shape covering the face region. All areas outside of the face AOIs were considered as the non-social background AOIs. To further investigate fixations to the face region, rectangular-shaped AOIs were designated at the eyes and mouth.

Two gaze parameters were analyzed: the fixation count - the number of fixations made inside an AOIand the total fixation duration - the addition of all fixation durations recorded within an AOI. A fixation event was defined as such by the tobii fixation filter when the point of gaze remained within 0.5 degree of visual angle for at least 100 milliseconds.

In order to investigate whether the detection of putative social perception abnormalities in autism is related to the type of stimuli presented, we have analyzed gaze parameters of children with ASD and control children during visualization of social scenes across four different types of stimuli. Since variation in gaze parameters absolute values in each AOI were observed in control subjects due to intrinsic characteristics of each stimulus (see Table 1), absolute values of gaze parameters were not appropriate for statistical analyses across stimuli. Therefore, the data obtained from the ASD group wes normalized into z-score values relative to the data obtained from the control group.

These normalized $z$-score values were analyzed using one-sample t-tests, and two-tailed $p$ values $<0.05$ were considered as abnormal gaze patterns in the ASD group. Because significant differences were only found for the fixation counts, further analyses were performed using only this parameter. To identify whether certain types of stimuli better discriminate the ASD group from the control group, we performed a two-way ANOVA using the ecological dimension [human actors/cartoon] and pres- 
entation form [movie/picture] in the four AOIs (eyes, mouth, face and non-social background) as factors.

\section{RESULTS}

Table 2 shows the normalized z-score values of fixation counts in the ASD group. Children from this group presented significantly fewer fixations to the eyes and face AOIs in the movie with human actors $(p<0.005$ and $p<$ 0.05 respectively) and picture with human actors $(\mathrm{p}<$ 0.005 and $\mathrm{p}<0.05$ respectively). Children with ASD also presented significantly more fixations to non-social background AOIs in both the movie with human actors $(\mathrm{p}<0.05)$ and the cartoon movie $(\mathrm{p}<0.05)$. In the cartoon movie, fixations were significantly decreased only in the mouth AOI in children with ASD $(\mathrm{p}<0.005)$. No significant differences were found between the two groups for the cartoon picture.

In the eyes AOI, the two-way ANOVA revealed a significant ecological effect [human actors $>$ cartoon] (F (1, $68)=5.94 ; \mathrm{p}=0.017)$ without a significant presentation effect $(\mathrm{F}(1,68)=0.71 ; \mathrm{p}=0.402)$ and without a significant interaction $(\mathrm{F}(1,68)=0.15 ; \mathrm{p}=0.288)$ (Figure 1(a)).

In the non-social AOI, the two-way ANOVA revealed a significant presentation form effect [movie $>$ picture] $(\mathrm{F}(1,68)=5.72 ; \mathrm{p}=0.019)$ without a significant ecological effect $(\mathrm{F}(1,68)=1.93 ; \mathrm{p}=0.169)$ and without a significant interaction $(F(1,68)=1.10 ; p=0.298)$ (Figure 1(b)).

Table 1. Control group data: number of fixations in each AOI in the four different stimuli.

\begin{tabular}{|c|c|c|c|c|}
\hline & Human actors movie mean & Human actors picture mean & Cartoon movie mean & Cartoon picture mean \\
\hline Non-social background & $1.71(1.49)$ & $2.19(2.42)$ & $1.38(0.59)$ & $1.95(2.36)$ \\
\hline Eyes & $4.0(2.19)$ & $6.57(3.19)$ & $2.91(1.84)$ & $5.62(2.38)$ \\
\hline Mouth & $1.19(1.36)$ & $2.19(1.57)$ & $1.67(1.43)$ & $1.48(1.12)$ \\
\hline Face & $7.14(2.56)$ & $10.14(3.20)$ & $6.29(1.71)$ & $8.14(2.52)$ \\
\hline
\end{tabular}

Table 2. Normalized z-score values of fixation counts in the ASD group and a statistical comparison to the control group.

\begin{tabular}{ccccc}
\hline & Human actors movie mean (SE) & Human actors picture mean (SE) & Cartoon movie mean (SE) & Cartoon picture mean (SE) \\
\hline Non-social background & $0.90(0.20)^{*}$ & $0.03(0.22)$ & $2.46(1.16)^{*}$ & $0.26(0.35)$ \\
Eyes & $-0.63(0.20)^{* *}$ & $-0.58(0.18)^{* *}$ & $0.14(0.26)$ & $-0.28(0.23)$ \\
Mouth & $-0.18(0.23)$ & $-0.05(0.30)$ & $-0.62(0.14)^{* *}$ & $0.02(0.29)$ \\
Face & $-0.40(0.19)^{*}$ & $-0.60(0.24)^{*}$ & $-0.23(0.31)$ & $-0.45(0.34)$ \\
\hline
\end{tabular}

${ }^{*} \mathrm{p} \leq 0.05 ;{ }^{* *} \mathrm{p} \leq 0.005$.

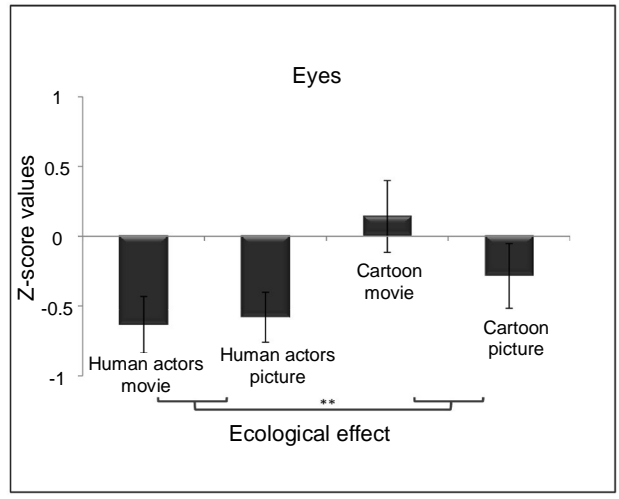

(a)

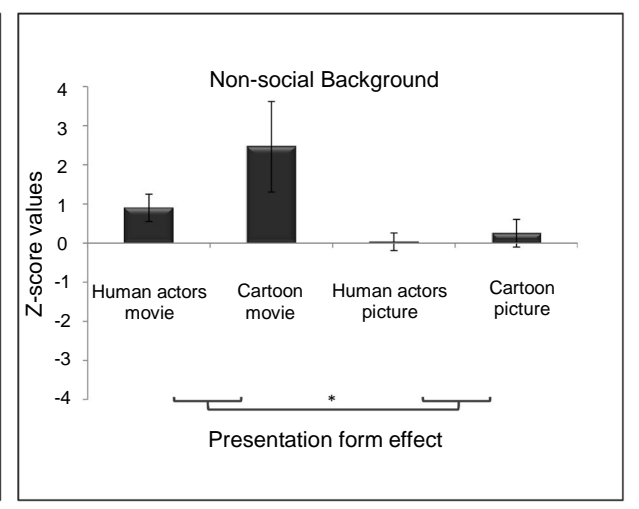

(b)

Figure 1. Analysis of stimulus effects on gaze parameters. (a) Ecological effect: Two-way ANOVA of z-score values for the eye AOI in the ASD group. This analysis showed that fixation to the eyes in the ecological conditions were significantly different from fixations to the eyes in the non-ecological conditions; (b) Presentation form effect: Two-way ANOVA of z-score values for the non-social background AOI in the ASD group. This analysis showed that fixations to the non-social background in the dynamic conditions were significantly different from fixation to the non-social background in the static conditions. 


\section{DISCUSSION}

The objective of this study was to investigate which of four different types of stimuli (movie with human actors, picture with human actors, cartoon movie and cartoon picture) would be more sensitive in detecting gaze abnormalities to better discriminate children with ASD from typically developing children. For that purpose, two different effects were tested: an ecological dimension effect [human actors/cartoon] and a presentation form effect [movie/picture].

A direct comparison of the four different types of stimuli confirmed the main hypothesis of this study that gaze pattern abnormalities depend on the type of stimuli. This dependency on the stimulus type may explain a certain extent of the heterogeneity observed in previous publications that have used eye-tracking to study social perception in autism.

As first main result of this study, a decrease in the number of fixations to the eyes was detected in children with ASD only in the ecological stimuli (movie and picture with human actors) but not in the cartoon stimuli (movie or picture) (Figure 2). Furthermore, the two-way ANOVA demonstrated a significant ecological effect. This analysis showed that number of fixations to the eyes in the ecological conditions was significantly different from number of fixations to the eyes in the non-ecological conditions. Therefore, ecological stimuli displaying human actors are more sensitive in detecting gaze abnormalities to the eyes in children with ASD than nonecological stimuli, such as cartoons.
The results concerning the ecological dimension effect corroborate most of the results from previous studies using human pictures $[9,16,17]$ or human actors movies $[10,12,14,15,18]$ that reported significantly diminished gaze to the eyes in subjects with autism when compared with typically developing controls. It is also supported by recent findings of identified abnormalities in gaze during naturalistic social interactions [22]. However, the present results disagree with results from some studies using human pictures as stimuli $[14,20]$, which found no significant differences between subjects with autism and typically developing controls and with the study by Nakano and colleagues [18], which used human actors movies and found no significantly diminished gaze to the eyes in children with ASD.

As second main result from this study, an increase in the number of fixations to the non-social background was detected in the ASD group only for dynamic stimuli (movie with human actors and cartoon movie) but not for static stimuli. In addition, the two-way ANOVA detected a significant presentation form effect. This analysis showed that number of fixations to non-social background in the dynamic conditions was significantly different from number of fixations to the non-social background in the static conditions. These results suggest that dynamic stimuli are more sensitive than static stimuli in detecting gaze abnormalities when concerning a preference for non-social features in a scene.

The results concerning the presentation form effect also confirm results from previous studies that used dy-

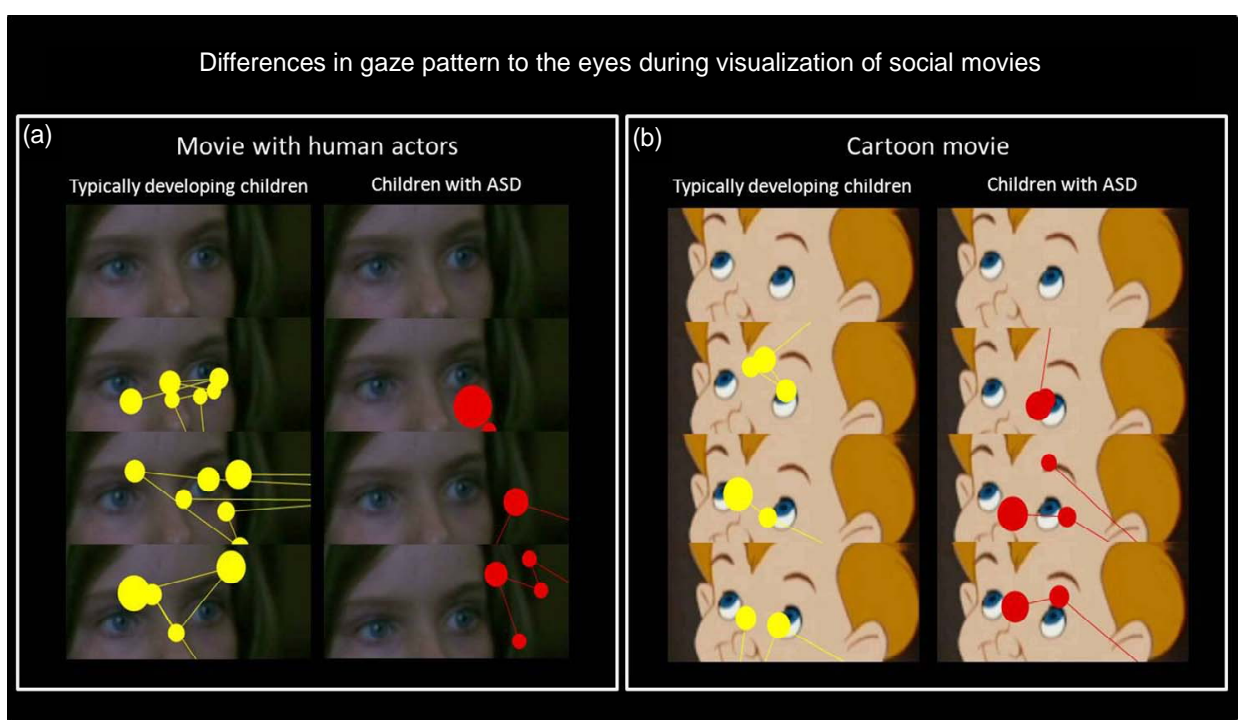

Figure 2. Differences in gaze pattern to the eyes during visualization of social movies. (a) Fixations to the eyes from three typically developing children and three children with autism when watching movie with human actors; (b) Fixations to the eyes from three typically developing children and three chil- dren with autism when watching cartoon movie. These results show that children with ASD, when compared to controls, look less to the eyes in the more ecologically relevant situation presenting hu- man actors. 
namic stimuli with human actors $[10,13]$ or cartoon characters [15] and reported significant differences between subjects with autism and typically developing controls with respect to gaze outside of social areas. The results of the present study also agree with results from studies that used static stimuli and found no significant differences between groups concerning gaze to the non-social background area $[9,14,20]$.

The results presented here suggest that general gaze abnormalities in children with autism are better detected when using dynamic stimuli. In addition, finer details of these abnormalities, especially looking less to the eyes, are only detected in a more ecologically relevant situation presenting human actors. Looking less to the eyes in an ecological situation may have a critical impact on the development of social skills in children with autism [25-27]. Since the eyes are a critical source of socially relevant information, if a child has less experience observing the eyes of others, this child is very likely to develop less expertise about social cues conveyed by the eyes, with cascading effects on further socialization. Such a capacity is a prerequisite for higher-level appreciation of the minds of others and is part of the larger cognitive domain of theory of mind and social cognition, which is severely impaired in autism.

When cartoon stimuli were used, the results showed that children with autism did not differ from control children with respect to gaze to the eyes. Interestingly, this observation may indicate that in this less ecological situation, children with autism can more easily access core social information from the eyes. If confirmed, this finding can support the development of new therapeutic strategies for improving social perception in children with autism.

\section{CONCLUSION}

Eye-tracking is becoming established as an important method in cognitive neuroscience research. For example, the use of eye-tracking in autism research can help to better characterize group phenotypes for brain imaging or genetic studies. In addition, eye-tracking studies could be used as an objective parameter to evaluate the impact of new therapeutic strategies in autism. For this reason, a precise methodology for social perception studies using eye-tracking in autism is necessary. Importantly, this study provides the first experimental evidence that detection of gaze abnormalities in ASD depend on the type of stimuli that is used.

\section{ACKNOWLEDGEMENTS}

We would like to acknowledge all children who took part in this study as well as their parents. We would also like to acknowledge the Association Les Amis d'Arthur for supporting the development of this re- search and Antoine Luu and Lépoldine Brand for consistent and permanent technical support.

\section{REFERENCES}

[1] Volkmar, F.R., Lord, C., Bailey, A., Schultz, R.T. and Klin, A. (2004) Autism and pervasive developmental disorders. Journal of Child Psychology and Psychiatry, 45, 135-170. doi:10.1046/j.0021-9630.2003.00317.x

[2] APA (1994) Diagnostic and statistical manual of mental disorders. 4th Edition, Washington DC.

[3] Leekam, S.R., Hunnisett, E. and Moore, C. (1998) Targets and cues: Gaze-following in children with autism. Journal of Child Psychology and Psychiatry, 39, 951962. doi:10.1111/1469-7610.00398

[4] Neumann, D., Spezio, M.L., Piven, J. and Adolphs, R. (2006) Looking you in the mouth: Abnormal gaze in autism resulting from impaired top-down modulation of visual attention. Social Cognitive and Affective Neuroscience, 1, 194-202. doi:10.1093/scan/ns1030

[5] Spezio, M.L., Huang, P.Y., Castelli, F. and Adolphs, R. (2007) Amygdala damage impairs eye contact during conversations with real people. Journal of Neuroscience, 27, 3994-3997. doi:10.1523/JNEUROSCI.3789-06.2007

[6] Levy, D.L., Sereno, A.B., Gooding, D.C. and O'Driscoll, G.A. (2010) Eye tracking dysfunction in schizophrenia: Characterization and pathophysiology. Current Topics in Behavioral Neurosciences, 4, 311-347. doi:10.1007/7854 2010 60

[7] Boraston, Z. and Blakemore, S.J. (2007) The application of eye-tracking technology in the study of autism. Journal of Physiology, 581, 893-898. doi:10.1113/jphysiol.2007.133587

[8] Gredeback, G., Johnson, S. and von Hofsten, C. (2010) Eye tracking in infancy research. Developmental Neuropsychology, 35, 1-19. doi:10.1080/87565640903325758

[9] Riby, D.M. and Hancock, P.J. (2008) Viewing it differently: social scene perception in Williams syndrome and autism. Neuropsychologia, 46, 2855-2860. doi:10.1016/j.neuropsychologia.2008.05.003

[10] Klin, A., Jones, W., Schultz, R., Volkmar, F. and Cohen, D. (2002) Visual fixation patterns during viewing of naturalistic social situations as predictors of social competence in individuals with autism. Archives of General Psychiatry, 59, 809-816. doi:10.1001/archpsyc.59.9.809

[11] Klin, A., Lin, D.J., Gorrindo, P., Ramsay, G. and Jones, W. (2009) Two-year-olds with autism orient to non-social contingencies rather than biological motion. Nature, 459, 257-261. doi:10.1038/nature07868

[12] Jones, W., Carr, K. and Klin, A. (2008) Absence of preferential looking to the eyes of approaching adults predicts level of social disability in 2-year-old toddlers with autism spectrum disorder. Archives of General Psychiatry, 65, 946-954. doi:10.1001/archpsyc.65.8.946

[13] Shic, F., Bradshaw, J., Klin, A., Scassellati, B. and Chawarska, K. (2011) Limited activity monitoring in toddlers with autism spectrum disorder. Brain Research, $\mathbf{1 3 8 0}$, 
246-254. doi:10.1016/j.brainres.2010.11.074

[14] Speer, L.L., Cook, A.E., McMahon, W.M. and Clark, E. (2007) Face processing in children with autism: Effects of stimulus contents and type. Autism, 11, 265-277. doi:10.1177/1362361307076925

[15] Riby, D. and Hancock, P.J. (2009) Looking at movies and cartoons: Eye-tracking evidence from Williams syndrome and autism. Journal of Intellectual Disability Research, 53, 169-181. doi:10.1111/j.1365-2788.2008.01142.x

[16] Pelphrey, K.A., Sasson, N.J., Reznick, J.S., Paul, G., Goldman, B.D., et al. (2002) Visual scanning of faces in autism. Journal of Autism and Developmental Disorders, 32, 249-261. doi:10.1023/A:1016374617369

[17] Dalton, K.M., Nacewicz, B.M., Johnstone, T., Schaefer, H.S., Gernsbacher, M.A., et al. (2005) Gaze fixation and the neural circuitry of face processing in autism. Nature Neuroscience, 8, 519-526.

[18] Nakano, T., Tanaka, K., Endo, Y., Yamane, Y., Yamamoto, T., et al. (2010) Atypical gaze patterns in children and adults with autism spectrum disorders dissociated from developmental changes in gaze behaviour. Proceedings of the Royal Society, 277, 2935-2943. doi:10.1098/rspb.2010.0587

[19] van der Geest, J.N., Kemner, C., Camfferman, G., Verbaten, M.N. and van Engeland, H. (2002) Looking at images with human figures: Comparison between autistic and normal children. Journal of Autism and Developmental Disorders, 32, 69-75. doi:10.1023/A:1014832420206

[20] van der Geest, J.N., Kemner, C., Verbaten, M.N. and van Engeland, H. (2002) Gaze behavior of children with pervasive developmental disorder toward human faces: A fixation time study. Journal of Child Psychology and Psychiatry, 43, 669-678. doi:10.1111/1469-7610.00055
[21] Falck-Ytter, T. and von Hofsten, C. (2011) How special is social looking in ASD: A review. Progress in Brain Research, 189, 209-222. doi:10.1016/B978-0-444-53884-0.00026-9

[22] Noris, B., Nadel, J., Barker, M., Hadjikhani, N. and Billard, A. (2012) Investigating gaze of children with ASD in naturalistic settings. PLoS One, 7, e44144. doi:10.1371/journal.pone.0044144

[23] APA (1994) Diagnostic and statistical manual of mental disorders. American Psychiatric Association, Washington DC.

[24] Boddaert, N., Zilbovicius, M., Philipe, A., Robel, L., Bourgeois, M., et al. (2009) MRI findings in 77 children with non-syndromic autistic disorder. PLoS One, 4, e4415. doi:10.1371/journal.pone.0004415

[25] Pelphrey, K.A., Morris, J.P. and McCarthy, G. (2004) Grasping the intentions of others: The perceived intentionality of an action influences activity in the superior temporal sulcus during social perception. Journal of Cognitive Neuroscience, 16, 1706-1716. doi: 10.1162/0898929042947900

[26] Klin, A., Jones, W., Schultz, R. and Volkmar, F. (2003) The enactive mind, or from actions to cognition: Lessons from autism. Philosophical Transactions of the Royal Society B: Biological Sciences, 358, 345-360. doi:10.1098/rstb.2002.1202

[27] Frith, U., Morton, J. and Leslie, A.M. (1991) The cognitive basis of a biological disorder: Autism. Trends in Neuroscience, 14, 433-438. doi:10.1016/0166-2236(91)90041-R 